\title{
Use of Relays in Extending Network Lifetime
}

\author{
Chen-Mou Cheng and H.T. Kung \\ Division of Engineering and Applied Sciences \\ Harvard University \\ Cambridge, MA, United States
}

\begin{abstract}
We describe a simple physical-layer relay scheme for wireless communication in which a set of low-noise linear amplify-and-forward relays are placed between transmitter and receiver to assist radio communication. By exploiting the geometric gain resulting from the location of properly deployed relays, part of the radio transmission power of the information source can be offloaded to these relays. We argue that such a simple relay scheme can thus provide a transparent and management-free alleviation to the problem of limited battery time in application scenarios like sensor networks, where radio transmission can consume a significant portion of the battery-provided energy. We use simulation to quantify the bit error rate (BER) performance of the relay scheme in low signal-to-noise (SNR) regime when used in conjunction with the Maximal Ratio Combining (MRC) scheme and the Alamouti space-time codes. We show that, under a reasonably precise indoor radio propagation model derived from measurements, the proposed relay scheme is capable of reaping the geometric gain attained from a set of relays deployed midway between the transmitter and the receiver to effectively extend the battery lifetime of the network.
\end{abstract}

\section{INTRODUCTION}

Power management is a great challenge in application scenarios like wireless sensor networks where energy is a scarce crucial resource. For instance, a small number of sensor nodes in a network may incur a relatively high energy consumption because of sensing activity or data processing, or because of inter-node or external communication. It is often the case that these power-demanding nodes can not be identified a priori, as they are situation-dependent. For example, when tracking a mobile target with an array of stationary sensors, those nodes which are closer to the current location of the target usually consume more power because of higher level of sensing and communication activities. When the battery power of these nodes runs out, the usefulness of the sensor network will be greatly reduced, for these nodes are precisely those which are required to perform the most critical operations for the application.

Given such a wireless sensor network where each node lives on a finite power source, we are interested in finding practical solutions that can extend the battery-limited lifetime of the network. Among the various power consuming components in such a node, the power used for radio transmission can constitute a significant portion of the total power consumption in many situations [5][6]. In this paper, we focus on this aspect and propose to alleviate the problem by deploying inexpensive and possibly disposable relays in the networking area to offload a major portion of the radio transmission power consumption on mission-critical nodes. Furthermore, the deployment of these relays can be "on-demand" in the sense that they can be added to areas which later on are determined to be in a powercritical situation after initial deployment of regular sensor/network nodes. In the future, it is also possible for these relays to take advantage of various emerging "energy-harvesting” technologies to renew their own energy using, e.g., solar, vibration, and/or other forms of natural energy sources [9], so that these relays can form a long-lasting wireless communication infrastructure that requires little or no maintenance.

The basic principle of the proposed approach is that, when communicating to a destination node, a source node can reduce its transmission power by transmitting signal to those relay nodes which are closer to the source node than the destination node is. These relay nodes then relay the received signal to the receiver using their own battery power. We will investigate the use of analog amplify-and-forward relays, i.e., devices which receive, amplify, and forward waveforms in analog regime without decoding and re-encoding. This results in not only a low-complexity circuit design but also a universal scheme that is transparent to communication system characteristics such as channel coding and modulation techniques at the two communication ends. As a result, the same cheap relays can be used in conjunction with many different communication systems.

The main role of these relays is to assist communication by being able to receive better signal because of being closer to the source node, and by amplifying the received signal to mitigate the noise associated with the next hop. It is important that such a relay does not introduce too much noise in the amplification process. Many state-of-the-art low-noise linear amplifiers satisfy this requirement [4].

Another issue faced by relay-assisted radio communication is that a relay can not receive and send radio signal at the same time on the same carrier frequency; otherwise the signal being transmitted will interfere with the signal being received. This is typically solved by using orthogonal channels provided by, e.g., time division multiplexing (TDM) or frequency divi- 
sion multiplexing (FDM) [7][8]. Thus, these relays will need to use at least two such orthogonal channels.

The rest of this paper is organized as follows. In Section II, we give a detailed description of the relay scheme and reason quantitatively why it can reduce radio transmission power at a source node. We present the BER performance simulation results in Section III, based on which we illustrate the potential power saving in Section IV. In Section V, we discuss some of the potential issues one might encounter during implementation and deployment of the relay scheme. We report related work in literature in Section VI and conclude this paper in Section VII.

\section{THE RELAY SCHEME}

In this section, we describe the basic relay scheme and give reasons why radio transmission power can be effectively offloaded from a source node.

Throughout the paper, we will use a simple scenario of Figure 1, which we call the model network configuration, to illustrate how the relay scheme works, as well as its various properties. Suppose that a source $\mathrm{S}$ and a destination $\mathrm{D}$ are two sensor nodes wishing to communicate with each other. We assume that relay nodes $\mathrm{R}_{\mathrm{i}}$ 's are situated at a location which, together with the locations of $\mathrm{S}$ and $\mathrm{D}$, form an equilateral triangle, as shown in Figure 1. Typically, $\mathrm{S}$ will start with the sending of a preamble signal, which may serve multiple purposes including marking the start of communication, facilitating synchronization, and/or providing information for channel estimation at $D$. Relay nodes $R_{1}$ through $R_{n}$ can be configured to detect this preamble signal and begin relay operation once triggered by such a signal. A similar mechanism or a time-outbased mechanism can be used to terminate the operation of relay nodes.

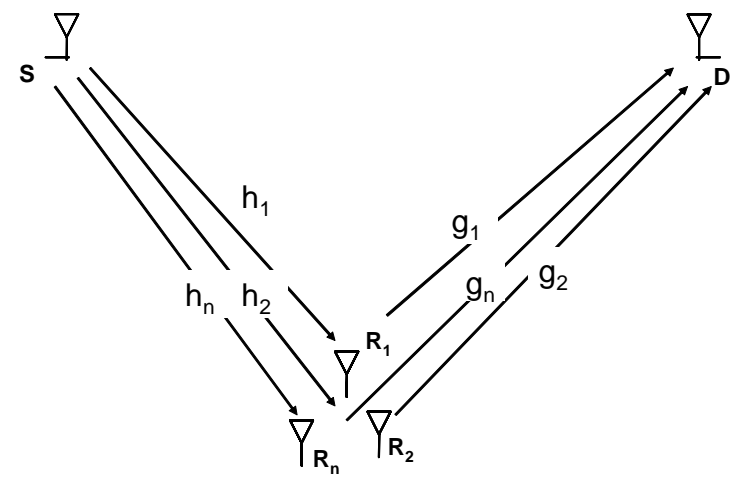

Figure 1. The model network configuration of this paper for illustrating the relay scheme. The source node $\mathrm{S}$ needs to stream a large amount of data to the destination node $\mathrm{D}$. The relay nodes $\mathrm{R}_{1}$ through $\mathrm{R}_{\mathrm{n}}$ are used to help offload the radio transmission power of $\mathrm{S}$
In Figure 1, the $h_{i}$ 's and $g_{i}$ 's represent the channel characteristics for the links $S-R_{i}$ and $R_{i}-D$, for $i=1, \ldots, n$. To model multipath propagation and fading, we adopt the flat Rayleigh fading channel model in this paper. That is, the fading amplitudes for all transmitter-receiver pairs are assumed to be mutually uncorrelated and Rayleigh distributed with a uniformly distributed phase shift within a period of time that is equal to or longer than the symbol time. Furthermore, fading amplitudes and phases are assumed to be uncorrelated across different periods of time.

We further assume that the synchronization problem at the $D$ with respect to arrivals from different $R_{i}$ 's is negligible. This means that the relay scheme is intended for transmission at moderate rates so that the corresponding symbol time is long enough to be tolerant to given synchronization imperfections.

We expect that the SNR observed at the $\mathrm{R}_{\mathrm{i}}$ 's will need to be higher than that at the $\mathrm{D}$ in order to achieve the same bit error rate (BER) for several reasons. First, since the relay nodes relay waveforms without restoring symbols, the noise from the first hop is also amplified and is no longer additive from D's point of view. Second, the noise from the second hop degrades communication quality when the total power used by relay nodes is not high enough. Lastly, both hops can suffer from fading, and the communication quality can not be easily improved when either hop is in deep fades in this simple scheme. Fortunately, as we will illustrate in Section IV, this penalty introduced by relaying in analog regime is usually smaller than the geometric gain resulting from the fact that some of the $\mathrm{R}_{\mathrm{i}}$ 's are closer to $\mathrm{S}$ than $\mathrm{D}$.

We now define several terms to facilitate quantitative reasoning about the relay scheme. Quantities such as gain and penalty usually depend on the underlying communication techniques, so first we need to fix a specific scheme; for example, in our simulation, we use Alamouti codes and coherent Binary Phase Shift Keying (BPSK) modulation. Consider a transmission from $\mathrm{S}$ to $\mathrm{D}$. We will consider two methods: the direct method, where $\mathrm{S}$ transmits to $\mathrm{D}$ directly without using any relay, and the relay method, where $\mathrm{S}$ transmits its signal to $\mathrm{D}$ through the relays $\mathrm{R}_{\mathrm{i}}$ 's.

We first define what we mean by the relay penalty. For a given BER, let snrd denote the required SNR at D in order to achieve the BER under the direct method. (The $d$ in snrd indicates that it is the SNR for the direct method.) Now consider the case when $\mathrm{S}$ transmits to $\mathrm{D}$ under the relay method. Let snr2 denote the SNR observed at D. That is, snr2 is the SNR for the hop from the $\mathrm{R}_{\mathrm{i}}$ 's to $\mathrm{D}$, the second hop of the relay method. Note that snr2 generally depends on the transmission power of the $\mathrm{R}_{\mathrm{i}}$ 's and their distance to $\mathrm{D}$. Given a BER and a (sufficiently large) snr2, let snr1 denote the required SNR for the hop from $\mathrm{S}$ to the $\mathrm{R}_{\mathrm{i}}$ 's, the first hop of the relay method, in 
order for the relay method to achieve the given BER. Note that snr1 depends on snr2, in the sense that if snr2 assumes a smaller value, then a larger snr1 will be required. However, if snr2 is below certain threshold (which depends on the given BER value), then the relay method can not achieve the given BER, no matter how large snr1 is. In this case, snr1 is undefined.

For the reasons mentioned previously, snr1 is larger than snrd. We define the relay penalty to be their difference:

$$
\text { RelayPenalty }=\text { snr } 1-\text { snrd }
$$

expressed in the units of decibels (dB). Note that the relay penalty should decrease when snr2 increases, but with a diminishing return.

In practice, when the $\mathrm{R}_{\mathrm{i}}$ 's are placed in-between $\mathrm{S}$ and $\mathrm{D}$, we expect that for the same transmission power of $S, R_{i}$ 's will observe a higher SNR than D (see, e.g., [2]). Equivalently, to achieve the same SNR, S can use a smaller transmission power to reach the $\mathrm{R}_{\mathrm{i}}$ 's than $\mathrm{D}$. Let $p$ and $q$ be the required transmission power of $\mathrm{S}$ in order to achieve the same SNR at $\mathrm{D}$ and the $\mathrm{R}_{\mathrm{i}}$ 's, respectively. We define the geometric gain to be the ratio of $p$ over $q$ :

$$
\text { GeometricGain }=\mathrm{p} / \mathrm{q}
$$

expressed in dB. Finally, we define the relay gain to the geometric gain minus the relay penalty, i.e.:

$$
\text { RelayGain = GeometricGain - RelayPenalty }
$$

Although, in this paper, we focus on single-layer relays, these definitions generalize to multiple-layer relays in a natural way.

\section{PERFORMANCE SiMUlATION}

In this section, we report BER performance simulation results, based upon which we will illustrate the relay gain and power saving for our model network configuration of Figure 1 in Section IV. We report the results for the $1 \mathrm{x} 4$ Maximal Ratio Combining (MRC) scheme and the 2x2 Alamouti space-time codes [1] under coherent BPSK modulation. Moreover, we focus on low-SNR regime for practicality consideration.

Figure 2 shows the BER performance for the relay approach for the case where snr1 is equal to snr2; this corresponds approximately to (e.g., the situation depicted in Figure 1) where the distance from $S$ to $R_{i}$ 's is equal to that from $R_{i}$ 's to $\mathrm{D}$, and the total power used by $\mathrm{R}_{\mathrm{i}}$ 's is equal to that used by $\mathrm{S}$. In addition to the $2 \times 2$ Alamouti space-time codes, we report the results for $1 \times 4$ MRC scheme. The latter might be of interest in situations where there are many cheap sensors and a stationary data collecting base station/control center, which can be equipped with a multi-element antenna to facilitate better reception of signal from sensors with single-element antennas.
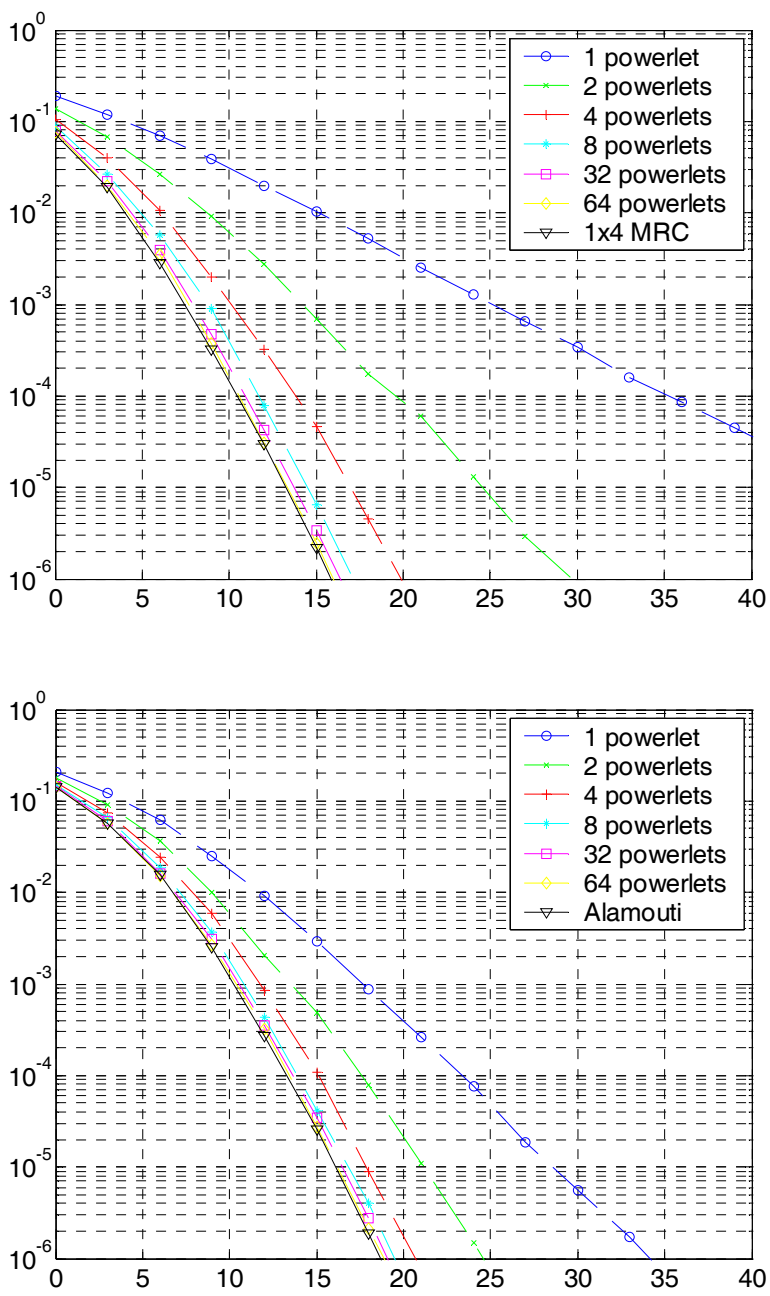

Figure 2. Performance of the relay scheme as a function of number of relay nodes used. Top: working in conjunction with the $1 \mathrm{x} 4$ Maximal Ratio Combining (MRC) scheme; Bottom: with the 2x2 Alamouti space-time codes

We see from Figure 2 that, with four or more relay nodes, the performance of the relay scheme is comparable to that of plain MRC or Alamouti scheme, i.e., the relay penalty is quite small. The reason why there should be sufficiently many relay nodes is to provide enough spatial diversity (uncorrelated channels) for space-time codes to work well.

We note that as more and more relay nodes are used, the power used per relay node decreases for achieving the same snr2 at the destination node D. With the advance in various energy harvesting technologies, we expect the gap between the amount of power provided by renewable energy sources and the amount of power needed by relays to be closing. One day we might even be able to see such relays be deployed ubiquitously to form an ambience-powered communication infrastructure ("wireless cables") to replace, say, today's Ethernet cables. 


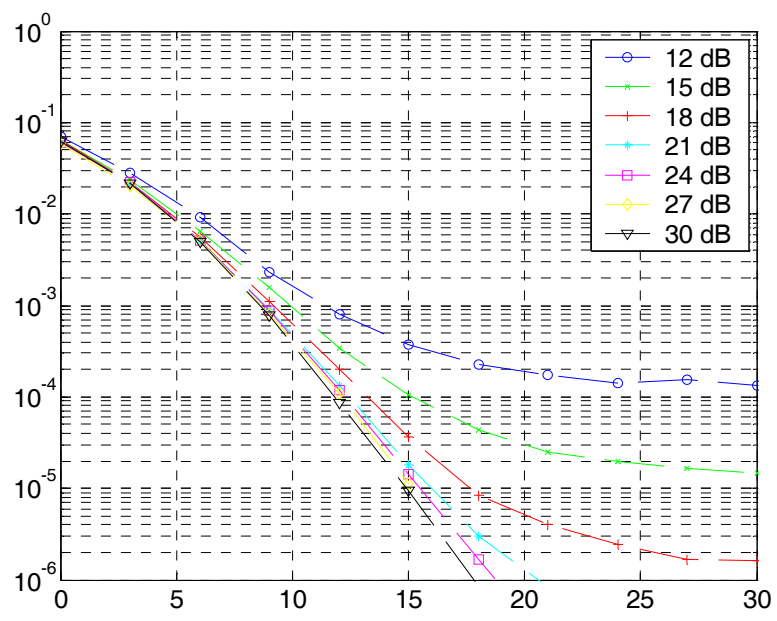

Figure 3. Performance of the relay scheme in terms of BER achieved by various snr 1 values, when four relays are used and when $s n r 2$ is assumed to be one of the several values shown

Figure 3 addresses a situation where snr2 remains constant while snr1 varies, i.e., the total output power of all relays is kept constant. The results show that for four relay nodes, when the $s n r 2$ reaches about $18 \mathrm{~dB}$, the performance is close to that of the plain Alamouti scheme that uses no relays, for BER in the range of $10^{-3}$ to $10^{-4}$.

\section{ILLUSTRATION OF RELAy GAIN AND POWER SAVING}

In this section, we give a simple calculation to demonstrate the potential relay gain achieved by the relay scheme. Our illustration will show that the relay gain can be significant, and thus the lifetime of the source node S can be substantially extended by using relays.

Consider, for example, the snr $2=18 \mathrm{~dB}$ case with four relays. In order for the relay scheme to achieve a BER of $10^{-4}$, we see from Figure 3 that $S$ 's transmission power is required to be such that the snr 1 is about $13 \mathrm{~dB}$. Note that from Figure 2 we see that to achieve the same BER for the plain Alamouti scheme, the required SNR (snrd) is about $10 \mathrm{~dB}$. This implies that the relay penalty is about $3 \mathrm{~dB}$, since $13 \mathrm{~dB}$ minus $10 \mathrm{~dB}$ is $3 \mathrm{~dB}$.

Using a measurement-based model in [2], we illustrate how the relay gain can be computed, and how it can result in power saving. Consider the scenario of Figure 1. Suppose that $\mathrm{S}$ wishes to stream to $\mathrm{D}$ a very large amount of video data at a certain data rate for as long as possible. To maintain an average BER of $10^{-4}$ with $2 \times 2$ Alamouti codes, $\mathrm{S}$ must use a certain amount of transmission power to maintain an average SNR from $\mathrm{S}$ to $\mathrm{D}$ (i.e., snrd) at $10 \mathrm{~dB}$ or above, according to the bot- tom half of Figure 2. Suppose that there are four relay nodes sitting midway between $\mathrm{S}$ and $\mathrm{D}$ as in the case of Figure 3 . Then, by the model of [2], the geometric gain is about $9 \mathrm{~dB}$. It follows that the relay gain is about $6 \mathrm{~dB}$, since, as noted above, the relay penalty is about $3 \mathrm{~dB}$. Note that $6 \mathrm{~dB}$ represents approximately a gain of factor four. This means that by using the relay method, $\mathrm{S}$ can stream to $\mathrm{D}$ at the same data rate with the same average BER as the direct method, but at only one quarter of the transmission power. If $\mathrm{S}$ is the power-critical node in the network, and if radio transmission power is the major source of power consumption, then the relay scheme will approximately quadruple the lifetime of the network.

It is instructive to observe from Figure 3 that for a given BER, the same increment in snr2 will result in more reduction in snr1 when snr2 is smaller. Consider, for example, the case when $\mathrm{BER}=10^{-5}$. A $3 \mathrm{~dB}$ increase in snr2 from $15 \mathrm{~dB}$ to 18 $\mathrm{dB}$ will reduce snr 1 by $9 \mathrm{~dB}$ (i.e., from $25 \mathrm{~dB}$ to $16 \mathrm{~dB}$ ), but the same $3 \mathrm{~dB}$ increase in snr2 from $18 \mathrm{~dB}$ to $21 \mathrm{~dB}$ will reduce snr1 only by $1 \mathrm{~dB}$ (i.e., from $16 \mathrm{~dB}$ to $15 \mathrm{~dB}$ ). This means that, for a given BER, there is a sweet spot in snr2 where an increase in the radio transmission power of the relay nodes is the most effective in minimizing snr1, or equivalently, the radio transmission power of $\mathrm{S}$.

\section{DISCUSSION}

In order to achieve optimal power saving for a given target BER, power control protocols such as those described in [11] can be used. We note that both the transmission power of the source node and that of the relay nodes need to be controlled. While the latter is a straightforward application of the existing feedback-based power control protocols, the former requires further investigation for the source node to obtain an accurate average SNR behavior of all participating relay nodes. This will be one of our research topics in the near future.

We also note that the new relay scheme might incur new security concerns. For example, a malicious user can trigger relay operations with little power and use the amplified signal to jam the frequency band at which relays operate. We can resort to physical-layer means to alleviate such a problem, e.g., by using techniques such as frequency hopping [14]. In addition, one can always raise the barrier for outside attackers by requiring the relay nodes to perform (some simple form of) authentication and authorization for triggering relay operations and setting up frequency hopping sequences. However, because the relays are simple analog devices, such authentication and authorization can be quite expensive to implement.

\section{RELATED WORK}

Wireless communication can seriously suffer from multipath fading. Many solutions to combat fading involve the use of some form of diversity, i.e., by transmitting in multiple logi- 
cal channels that fade independently from one another. This usually requires multiple antenna elements be installed at the two communication ends [3]. However, in low-cost wireless devices such as sensors, this approach may not be very attractive due to hardware limitation. Instead, it is desirable if singleantenna devices can dynamically pool together and share their antennas to form a distributed, cooperative antenna array [10].

In [12][13], a scheme is proposed in which users of a CDMA system cooperate with one another to exploit spatial diversity. Roughly speaking, each user will try to decode the partner's previously transmitted message and forward his/her best estimate of the message to the base station. The power is allocated according to the water pouring principle, i.e., more power is allocated when the channel state is favorable.

Work in [7][8] investigates the performance of amplifyand-forward cooperative schemes and finds that they converge asymptotically to that of decode-and-forward relay scheme. In addition, a relay protocol is proposed that can be regarded as being an approximation to the optimal power allocation scheme, i.e., a relay simply stops relaying when the channel is suffering deep fades and hence becomes worse than the channel of direction transmission.

To our best knowledge, all existing works assume symmetric roles among cooperative nodes. Our work differs in that we seek to build low-complexity power modules that aim to offload the radio transmission power of an information source node in a distributed manner. Also, we are more interested in the low-SNR BER performance rather than asymptotic trends for practical reasons.

\section{CONCLUSION}

We have described a simple physical-layer scheme where low-complexity relays can offload a major portion of the transmission power demands from mission-critical nodes, thereby effectively extending network lifetime. The relay scheme incurs very little management or protocol overheads. The main contributions of this paper are: identifying the use of transparent, low-complexity relays in extending network lifetime, quantifying the BER performance of the relay scheme in lowSNR regime, and exploiting the geometric gain attained under a realistic radio propagation model for power saving.

\section{ACKNOWLEDGEMENTS}

This research was supported in part by NSF grant \#ACI0330244, and in parts by research grants from Microsoft Research and Sun Microsystems.

\section{REFERENCES}

[1] Alamouti, S. M., "A simple transmit diversity technique for wireless communication," IEEE Journal on Select Areas in Communications, vol. 16, no. 8, Oct. 1998.
[2] Ghassemzadeh, S. S., L. J. Greenstein, A. Kavcic, T. Sveinsson, and V. Tarokh, "UWB indoor delay profile model for residential and commercial buildings,” in Proc. IEEE VTC2003-Fall, Orlando, FL, Oct. 2003.

[3] Gesbert, D., M. Shafi, D. Shiu, P. J. Smith, and A. Naguib, "From theory to practice: An overview of MIMO space-time coded wireless systems," IEEE Journal on Selected Areas in Communications, vol. 21, no. 3, Apr. 2003.

[4] Goo, J.-S., H.-T. Ahn, D. J. Ladwig, Z. Yu, T. H. Lee, and R. W. Dutton, "A noise optimization technique for integrated low-noise amplifiers," IEEE Journal of Solid-State Circuits, vol. 37, no. 8, Aug. 2002.

[5] Juang, P., H. Oki, Y. Wang, M. Martonosi, L. S. Peh, D. Rubenstein, "Energy-efficient computing for wildlife tracking: design tradeoffs and early experiences with ZebraNet," in Proc. ACM ASPLOS-X, San Jose, CA, Oct. 2002.

[6] Kahn, J. M., R. H. Katz, and K. S. J. Pister, "Next century challenges: mobile networking for 'Smart Dust,'” in Proc. ACM/IEEE MobiCom '99, Seattle, WA, Aug. 1999.

[7] Laneman, J. N., G. W. Wornell, and D. N. C. Tse, "An efficient protocol for realizing cooperative diversity in wireless networks," in Proc. IEEE ISIT 2001, Washington, DC, Jun. 2001.

[8] Laneman, J. N., and G. W. Wornell, "Distributed space-time-coded protocols for exploiting cooperative diversity in wireless networks," IEEE Trans. Information Theory, vol. 49, no. 10, Oct. 2003.

[9] Mannion, P., “'Energy harvesting' brings power to wireless nets,” EE Times, Oct. 2003.

[10] Nosratinia A., T. E. Hunter, and A. Hedayat, "Cooperative communication in wireless networks," IEEE Communications Magazine, accepted for publication.

[11] Novakovic, D. and M. Dukic, "Evolution of the power control techniques for DS-CDMA toward 3G wireless communication systems," IEEE Communications Surveys \& Tutorials, vol. 3, no. 4, 2000.

[12] Sendonaris, A., E. Erkip, and B. Aazhang, "User cooperative diversityPart I: System description,” IEEE Trans. Communications, vol. 51, no. 11, Nov. 2003.

[13] Sendonaris, A., E. Erkip, and B. Aazhang, "User cooperative diversityPart II: Implementation aspects and performance analysis,” IEEE Trans. Communications, vol. 51, no. 11, Nov. 2003.

[14] Zander, J., "Adaptive frequency hopping in HF communications," in Proc. IEEE MilCom ‘93, Boston, MA, Oct. 1993. 